\title{
Extracorporeal Cardio-Pulmonary Resuscitation (E-CPR) in Traumatic Cardiac Arrests Caused by Penetrating Thoracic Injuries: A Series of Two Cases
}

\author{
Viktor A Reva MD PhD', David T McGreevy MD², Eduard A Sinyavskiy MD PhD', \\ Daniil A Shelukhin MD PhD³, Alexander N Petrov MD PhD', \\ Alexander A Rud' MD PhD', Evgeniy N Ershov MD PhD', \\ Grigory E Lysenko $\mathrm{MD}^{4}$ and Igor M Samokhvalov MD PhD ${ }^{1}$ \\ 'Department of War Surgery, Kirov Military Medical Academy, Saint-Petersburg, Russia \\ ${ }^{2}$ Department of Cardiothoracic and Vascular Surgery, Örebro University Hospital, Örebro, Sweden \\ ${ }^{3}$ Nikiforov Russian Center of Emergency and Radiation Medicine, Saint-Petersburg, Russia \\ ${ }^{4}$ Department of Anesthesiology and Reanimatology, Kirov Military Medical Academy, Saint-Petersburg, Russia
}

\begin{abstract}
Background: We present two cases of thoracic penetrating injuries that necessitated extracorporeal cardiopulmonary resuscitation (E-CPR).

Methods: Two male patients were admitted to hospital within 20-25 min: one with a chest stab wound and the other with a gunshot injury. Upon ongoing CPR, patient \#1 underwent resuscitative sternotomy. Bleeding from a right ventricle injury was controlled, but cardiac arrest (CA) re-occurred. Patient \#2 underwent immediate surgery due to multiple rib fractures and massive hemopneumothorax, and experienced multiple CAs. Due to refractory asystole with ongoing CPR, extracorporeal membrane oxygenation (ECMO) was initiated after 100 and 135 min, respectively. Primary lactate levels in cases \#1 and \#2 were 8 and $20 \mathrm{mmol} / \mathrm{L}$, respectively.

Results: In both cases, the femoral artery (17-19 Fr) and vein (25-27 Fr) were cannulated and connected to the Maquet ECMO circuit with a flow rate of 4-5 L/min. Return of spontaneous circulation was achieved within 20 min after ECMO initiation with relative stabilization. In patient \#1, postoperative bleeding necessitated re-thoracotomy and hemorrhage control. In patient \#2, left pulmonectomy and ligation of intercostal arteries was performed. $12 / 30$ units of red blood cells, 16/45 units of fresh frozen plasma, and 2/8 units of platelets were given in cases $\# 1 / 2$, respectively. Lactate level increased to $25 \mathrm{mmol} / \mathrm{L}$ and decreased to $8 \mathrm{mmol} / \mathrm{L}$ in 5 hours, respectively. Both patients died in the ICU within 9 and 13 hours after admission due to bleeding.

Conclusions: E-CPR allows vital function protection even in traumatic CA but necessitates appropriate resuscitation. If no bleeding control is achieved, then E-CPR is futile .
\end{abstract}

Keywords: Thoracic Injury; ECMO; Cardio-Pulmonary Resuscitation; Cannulation; Endovascular Trauma Management

\section{Corresponding author:}

Viktor A. Reva, Department of War Surgery, Kirov Military Medical Academy, 6 Lebedeva str. Saint-Petersburg, Russian Federation 194044.

Email:vreva@mail.ru

Presentation: This work was presented as a poster at the Endovascular Hybrid Trauma and Bleeding Management (EVTM) symposium, November 16-17, 2019, in Denver, USA.

(C) 2020 CC BY 4.0 - in cooperation with Depts. of Cardiothoracic/ Vascular Surgery, General Surgery and Anesthesia, Örebro University Hospital and Örebro University, Sweden

\section{INTRODUCTION}

Penetrating thoracic injury remains one of the main causes of pre-hospital and early in-hospital cardiac arrest (CA) and death. Sudden deterioration can be due to either hemorrhagic or obstructive shock. The former results from uncontrolled massive bleeding into a pleural cavity, while the latter is caused by cardiac injury and intrapericardial bleeding followed by cardiac tamponade.

In the case of sudden CA caused by a penetrating thoracic injury, early resuscitative thoracotomy is considered the best option to release tamponade, cross-clamp the 
aorta, and/or control bleeding. Without appropriate surgery, a standard protocol of cardio-pulmonary resuscitation (CPR) is often futile and the chance of survival in traumatic CA (TCA) is diminished. A source of hemodynamic instability has to be addressed early and controlled to maintain circulation volume and organ perfusion.

A method of veno-arterial extracorporeal membrane oxygenation (V-A ECMO) or extracorporeal CPR (E-CPR) has been proposed and implemented into clinical practice to give artificial circulatory support during non-traumatic CA [1]. It is now widely used even for out-of-hospital medical CA by emergency ECMO teams in some developed countries [1-3].

E-CPR, however, has some significant limitations in trauma associated with uncontrolled bleeding and the extensive heparin administration used to maintain the ECMO circuit. Its use in TCA is still controversial because a trauma patient has a high risk of associated traumatic brain injury, acute coagulopathy, and may have hidden hemorrhagic loci. However, some promising reports of successful V-A ECMO use in polytrauma and bleeding shock have recently been published in the literature [4-6]. While these papers describe predominantly blunt trauma, data concerning E-CPR use in penetrating injuries is lacking [6].

We present two cases of penetrating thoracic injuries with attempted E-CPR that led to intermittent return of spontaneous circulation (ROSC) but ultimately failed to restore perfusion.

\section{Overview}

The two patients suffered penetrating thoracic trauma but underwent surgery in different environments. The first patient (case \#1) was stabbed in close proximity to a designated level 1 trauma center, while the second patient (case \#2) received an injury from a sawn-off shotgun within a public hospital that is not a trauma center and provides only elective daily surgery. In these two cases, different door-to-surgery times but similar time to initiation of ECMO were recorded. Experienced specialists and advanced technology were used similarly in both cases. No ultrasound was used to evaluate cardiac activity.

\section{Description of Cases}

\section{Case \#1}

A 25-year-old man was admitted with ongoing CPR to a trauma center within $20 \mathrm{~min}$ of receiving a midline chest stab injury at the base of the xyphoid process. Before admission, he was intubated, closed cardiac massage was initiated, but no intravenous (IV) access was achieved. The patient received $100 \%$ oxygen and monitoring showed pulseless electrical activity. Chest compressions were delivered using a mechanical device (Lucas $2^{\circledR}$,
Sweden) and the patient was immediately transported directly to an operation room (OR), bypassing the emergency department. An intraosseous needle (Novoplast$\mathrm{M}^{\circledR}$, Russia) was injected into the tibial plateau for fluid replacement therapy and surgery was initiated.

After removal of Lucas $2^{\circledR}$, an immediate median sternotomy was performed to release cardiac tamponade and a through-and-through right ventricle injury was sutured followed by open cardiac massage (OCM; Figure 1). Both pleural cavities were simultaneously drained. Abdominal focused assessment with sonography for trauma (FAST) was negative. Aggressive administration of fluids and inotropes through established large-bore central IV-lines along with OCM resulted in a temporary ROSC followed by repeated CAs and fibrillations (6 times), requiring electrical defibrillations and OCM. Arterial blood gases (ABG) within 60 min after admission demonstrated severe acidosis: $\mathrm{pH}$ 6.996; base excess $-19 \mathrm{mmol} / \mathrm{L}$; lactate $8 \mathrm{mmol} / \mathrm{L}$; partial pressure of oxygen $\left(\mathrm{pO}_{2}\right) 251 \mathrm{mmHg}$; partial pressure of carbon dioxide $\left(\mathrm{pCO}_{2}\right) 49.8 \mathrm{mmHg}$; oxygen saturation $\left(\mathrm{sO}_{2}\right)$ 99\%; hemoglobin $7 \mathrm{~g} / \mathrm{dL}$; and ROTEM demonstrated severe coagulopathy (internal thromboelastometry (INTEM): clotting time CT $750 \mathrm{~s}$ [normal range, (NR) 100-240]; external thromboelastometry (EXTEM): CT 151 s [NR 38-79 s], $\alpha$ angle $33^{\circ}$ [NR 70 $-83^{\circ}$ ], Amplitude at $10 \mathrm{~min}$ (A10) $7 \mathrm{~mm}$ [NR 43-65], no other parameters were detected by the machine).

During the resuscitative efforts, including blood transfusions and CPR, secondary FAST examination showed free fluid in the abdomen that necessitated a midline laparotomy. Superficial liver lacerations were found and tight perihepatic packing was effectively performed. Shed blood from both thoracic and abdominal cavities was collected using a Haemonetics Cell Saver ${ }^{\circledR}$ 5+ Autotransfusion System (USA) and subsequently re-infused $(7160 \mathrm{~mL})$.

After one hour of intermittently effective resuscitative efforts requiring repeated short-term aortic crossclamping via an additional left lateral incision (supraceliac clamping was avoided due to perihepatic packing), the decision was made to initiate ECMO to maintain perfusion of vital organs. Within 100 min after admission, the unilateral (right) femoral artery and vein were cannulated in an open fashion (the semi-Seldinger technique) by an ECMO team using 17-Fr and 25-Fr cannulas, respectively, and connected to the Cardiohelp ECMO (Maquet, Germany) circuit with a flow rate of $4-5 \mathrm{~L} / \mathrm{min}$. ROSC was achieved $28 \mathrm{~min}$ after ECMO initiation ( 2 hours after hospital admission) with relative stabilization of mean arterial pressure at 50-60 mmHg.

At this stage, $\mathrm{ABG}$ had improved: $\mathrm{pH} 7.263$; base excess -11 mmol/L; $\mathrm{pO}_{2} 421 \mathrm{mmHg}$; pCO $33.4 \mathrm{mmHg}$ $\mathrm{sO}_{2} 100 \%$; hemoglobin $8 \mathrm{~g} / \mathrm{dL}$; platelets $45 \times 10^{9} / \mathrm{L}$. However, the ionized calcium level decreased from 0.61 to $0.47 \mathrm{mmol} / \mathrm{L}$ despite calcium chloride administration. 


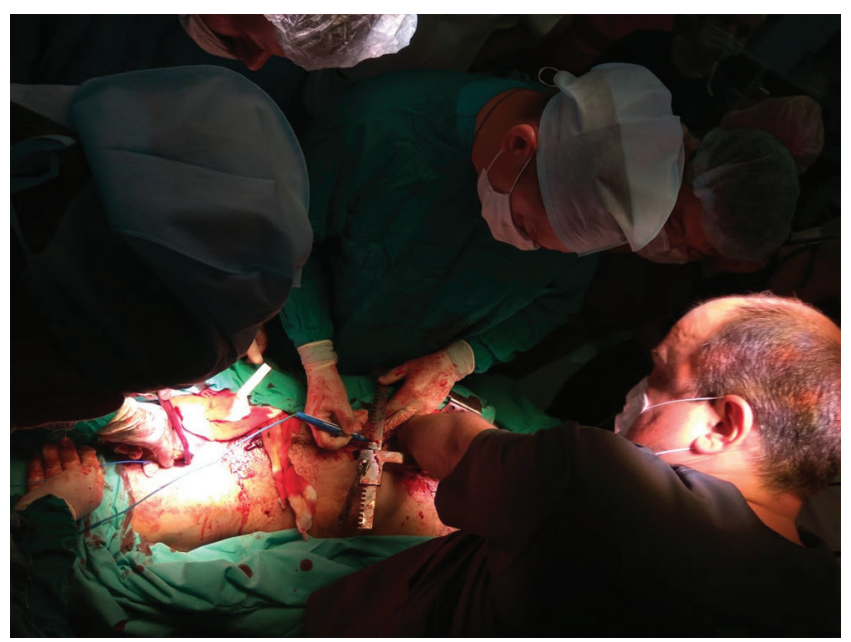

Figure 1 Emergency sternotomy and open cardiac massage along with an open femoral cutdown (for fluid replacement followed by extracorporeal membrane oxygenation cannulation) performed on patient \#1 admitted with ongoing cardio-pulmonary resuscitation.

Intraoperatively, in addition to autologous transfusion, the patient received 12 units of red blood cells (RBCs), 16 units of fresh frozen plasma (FFP), and 2 units of platelets. At the end of surgery his lactate level worsened to $25 \mathrm{mmol} / \mathrm{L}$ and ROTEM continued to demonstrate hypocoagulation (INTEM: CT 1099 s, $\alpha$ angle $12^{\circ}$, A10 9 mm, (Lysis Index after $30 \mathrm{~min}$ ) LI30 100\% [NR 94-100\%]; EXTEM: CT 110 s, $\alpha$ angle 14º A10 $12 \mathrm{~mm}, \mathrm{LI} 30$ 100\%). Clinically, postoperative bleeding from the thoracotomy wound occurred that necessitated re-thoracotomy and hemorrhage control.

The patient was referred to the intensive care unit (ICU) where he continued to deteriorate with permanent CA, a decrease in blood pressure despite extracorporeal life support, and limited passive movements in the joints of both legs. Further care was deemed futile and discontinued within 9 hours after admission.

\section{Case \#2}

A 61-year-old deputy medical director of a public hospital was assaulted by an agitated patient and suffered a gunshot injury to his left chest. He was found pale, pulseless, breathless, and unresponsive in his office by medical staff immediately after being shot. External CPR was initiated; definitive airway followed by bag ventilation was achieved before transport to an OR. Within 8 min after injury, the patient underwent resuscitative left antero-lateral thoracotomy through the fifth intercostal space. Approximately $2 \mathrm{~L}$ of fresh and clotted blood were evacuated from the pleural cavity. Injuries had been sustained to multiple left lung, intercostal, and left internal thoracic arteries. No cardiac injury was found after pericardiotomy and exposure of the arrested heart. OCM was initiated. For adequate fluid resuscitation, open cutdown access to both femoral veins was achieved followed by cannulation and massive fluid replacement therapy. Bleeding from multiple lung wounds re-occurred, were difficult to control, and necessitated left total pneumonectomy using a linear stapler (Ethicon Inc., USA); injured intercostal arteries were ligated. A cardiac surgeon and an ECMO team were called for a suspected blunt cardiac injury with development of cardiogenic death. A femoral artery was cannulated for intra-aortic balloon pump (IABP) insertion, and a temporary cardiac pacer was implanted. These efforts resulted in a temporary ROSC. Intermittent cardiac massage and defibrillations (19 times in total) along with attempts at hemorrhage control lasted for 2 hours. Diffuse bleeding from the ruptured muscles and vessels from the back of the pleural cavity was controlled by tight packing. Due to refractory asystole, the left femoral artery (19 Fr) and vein (an upsize from a previously inserted catheter to a 27-Fr ECMO cannula) were cannulated via the cutdown access and connected to the Rotaflow ECMO (Maquet, Germany) circuit with a flow rate of $4 \mathrm{~L} / \mathrm{min}$, $135 \mathrm{~min}$ after injury (Figure 2). Aggressive transfusion with 30 units of RBCs, 45 units of FFP and 8 units of platelets, and ECMO support resulted in a decrease in lactate level from $20 \mathrm{mmol} / \mathrm{L}$ upon admission to $8 \mathrm{mmol} / \mathrm{L}$, and relative stabilization of systolic BP from around 40-50 mmHg to more than $80 \mathrm{mmHg}$. After 7 hours of surgery, the patient was referred to an ICU where he continued to deteriorate and, 13 hours after injury, care was deemed futile and withdrawn.

\section{Ethical Approval and Informed Consent}

Ethical approval was not required. Informed consent was not possible because of a clear, immediate and serious threat to patients' life and the information has been anonymized.

\section{DISCUSSION}

E-CPR with ECMO is a sophisticated treatment option which may improve outcomes in a selected patient population and, when compared with conventional CPR, yields more neurologically intact survivors [7]. Data published on the use of E-CPR is still limited and its use is often part of an aggressive approach to resuscitation. E-CPR in the trauma population remains controversial and viewed by many as challenging with the risk of hemorrhage, especially in cases of severe coagulopathy, contraindications to anticoagulant use, or traumatic brain injury [8]. Nevertheless, recent growing evidence suggests that applying ECMO as a rescue therapy in trauma patients may provide potential survival benefits [9].

Both patients in this case report suffered from TCA following penetrating thoracic trauma. They were both quickly taken to an OR and received CPR with repeated defibrillations and massive transfusion, and V-A ECMO 


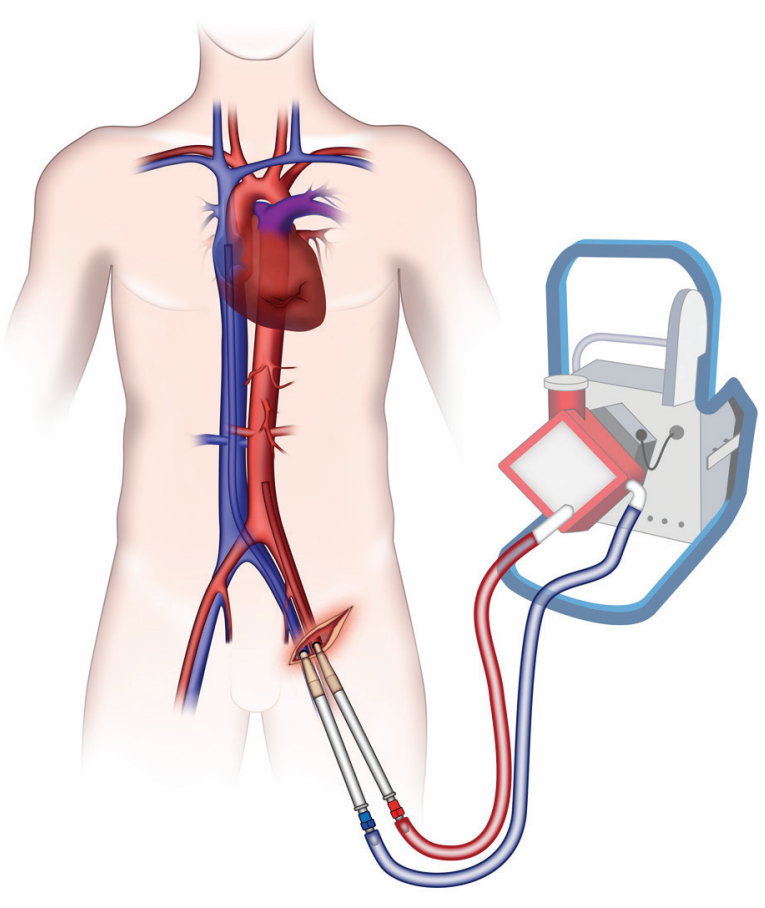

Figure 2 Schematic illustration of the extracorporeal membrane oxygenation circuit and open cannulation technique used in our patients.

was initiated within 120-135 min. However, the ECMO programs in these two hospitals differ. The patient in case \#1 was taken to a level 1 trauma center and initially submitted to aortic cross clamping. This hospital has the capacity for ECMO on-site including the machine, instruments, and specialists available 24/7. Whereas in case \#2, the ECMO team had to be called to the patient who received an IABP while waiting. According to the city program, this ECMO team is based at one hospital serving a whole area and has a fully equipped "ECMO-mobile" available 24/7 to be transferred to small hospitals if required.

It could be argued that resuscitative endovascular balloon occlusion of the aorta (REBOA) possibly should have been considered in both patients, if placed early during CPR and used as a bridge to ECMO; however, the use of REBOA in penetrating thoracic injury is currently considered to be contraindicated. While a REBOA-catheter was unavailable in case \#2, an attempt to support hemodynamics by using the IABP was undertaken. The underlying reason was also to increase postload if blunt cardiac injury (by shock waves) took place. Other bridging techniques for severely unstable or even arrested patients have been described in the literature, such as selective aortic arch perfusion [10] and different mechanical devices, e.g. Impella (Abiomed Inc., USA) and others, that can be more suitable options as a bridge to ECMO [11]. Right-sided Impella $\mathrm{RP}^{\circledR}$ could, for example, minimize the effect of left-sided pneumonectomy (case \#2) by removing blood from the inferior vena cava and ejecting it into the pulmonary artery, thus avoiding right heart failure. However, all these devices are very expensive and require fluoroscopy guidance for correct placement.

Placing the patients on ECMO allowed for the stabilization of blood pressure and relieved the heart by unloading the right atrium and ventricle, which intermittently resulted in periods of ROSC. In both cases, a high blood flow rate of 4-5 L/min was used to reduce the risk of blood clotting in the ECMO device since appropriate anti-coagulation to prevent thrombosis is contraindicated in trauma patients who are already coagulopathic as it might cause, or further contribute, to severe hemorrhage. Avoiding the use of heparin was particularly important in case \#2 as attempts at hemorrhage control lasted several hours. It is, however, important to remember the severe coagulopathy that occurs after trauma and cardiac arrest with a profound alteration of hemostasis and risk of developing organ dysfunction. Cardiac arrest with the acute lack of pulsatile blood flow facilitates rapid clot formation. After CPR and ROSC there is a brief period of systemic fibrinolysis that is later replaced by a prolonged hypercoagulable state. Trauma with tissue injury causes the release of factors that are responsible for coagulopathy, fibrinolysis, and activation of inflammatory pathways. This is important during ECMO treatment and therefore continuous monitoring of prothrombin time, international normalized ratio, and activated partial thromboplastin time is necessary throughout the procedure. The balance between minimizing the risk of surgical hemorrhage by not administering anticoagulants and the risk of an ECMO circuit thrombosis due to inadequate anticoagulation is always challenging, especially in severe trauma [12]. Despite aggressive resuscitation, both patients remained in hypocoagulation status. For better control of coagulation, viscoelastic assays, e.g. thromboelastography or ROTEM, have been proposed and are now widely used for patients with hemorrhages [13]. Low EXTEM clotting time is associated with an increased bleeding tendency and indicates a likely factor deficiency. Fibrinogen is the first coagulation factor that reaches critically low levels during major hemorrhage and remains low during ECMO. The administration of tranexamic acid is regularly used in trauma because of its antifibrinolytic effect and administration can be continued with signs of bleeding during ECMO support. In case \#1, ROTEM demonstrated a certain improvement over the course of treatment, with fibrinolysis at least being controlled (LI 30 turned to NR in a few hours). In case \#2, however, no coagulation monitoring was available at the treatment facility.

ECMO clearly plays an important role in trauma patients; however, evidence is still lacking. The first large multicenter report of trauma patients treated with ECMO by Swol et al. [7] showed a favorable survival rate of $61 \%$. Unfortunately, in the cases presented here, 
both patients finally deceased as a result of failure to restore perfusion within an adequate time period and may have benefited from being started on ECMO earlier in the course of treatment. It is important that the decision to place a patient on ECMO is made early, as time from decision to initiation of treatment takes on average 20-30 min if the treating facility has an ECMO team on-site. In addition to an early decision to start ECMO, a few other factors can theoretically improve survival in these critically unstable patients: a dedicated team readily available 24/7, including a cardiac surgeon, anesthetist, and perfusionist; a loaded ECMO set of instruments, lines and cannulas in the emergency department; extensive monitoring; and a free approach to blood and components (even whole blood), coagulation factors, etc. The decision to start ECMO is made by both anesthetist and surgeon based on vital signs upon admission and duration of CPR. Refractory CA, defined as 10-30 min of CPR without ROSC, can be an indicator for when ECMO might be implemented. Although optimal duration of CPR for successful ROSC is unknown, Lee et al. reported a case of successful ECMO combined with hypothermia in an arrested patient who suffered from a penetrating cardiac injury previously treated with 10 to $20 \mathrm{~min}$ of CPR [14]. Once the decision to start ECMO is made, the initiation of the procedure should begin as early as possible. Further studies are warranted to define indications for ECMO in TCA, particularly in chest injuries.

\section{CONCLUSION}

This case report demonstrates that ROSC can be achieved in patients with penetrating traumatic injuries and TCA with the use of ECMO without the administration of heparin. It is becoming increasingly clear that E-CPR with ECMO plays an important role in the treatment of trauma patients, but the exact role that it should play is as yet unknown and further investigation is therefore needed.

\section{Acknowledgements}

The authors thank all the surgeons and anesthetists who took care of these patients.

\section{Ethics Statement}

(1) All the authors mentioned in the manuscript have agreed to authorship, read and approved the manuscript, and given consent for submission and subsequent publication of the manuscript.

(2) The authors declare that they have read and abided by the JEVTM statement of ethical standards including rules of informed consent and ethical committee approval as stated in the article.

\section{Conflicts of Interest}

The authors declare that they have no conflicts of interest.

\section{Funding}

The authors received no financial support for the research, authorship, and/or publication of this article.

\section{Author Contributions}

VAR, DM, and EAS carried out the study design, data interpretation, and manuscript writing. DAS, AAR, ANE, and GEL perfromed data analysis and interpretation. IMS and ANP carried out critical revision and work supervision.

\section{REFERENCES}

[1] Hutin A, Abu-Habsa M, Burns B, et al. Early ECPR for out-of-hospital cardiac arrest: Best practice in 2018. Resuscitation. 2018;130:44-8.

[2] Maekawa K, Tanno K, Hase M, Mori K, Asai Y. Extracorporeal cardiopulmonary resuscitation for patients with out-of-hospital cardiac arrest of cardiac origin: a propensity-matched study and predictor analysis. Crit Care Med. 2013;41(5):1186-96.

[3] Lamhaut L, Hutin A, Puymirat E, et al. A pre-hospital extracorporeal cardio pulmonary resuscitation (ECPR) strategy for treatment of refractory out hospital cardiac arrest: an observational study and propensity analysis. Resuscitation. 2017;117:109-17.

[4] Bonacchi M, Spina R, Torracchi L, Harmelin G, Sani G, Peris A. Extracorporeal life support in patients with severe trauma: an advanced treatment strategy for refractory clinical settings. J Thorac Cardiovasc Surg. 2013;145(6):1617-26.

[5] Arlt M, Philipp A, Voelkel S, et al. Extracorporeal membrane oxygenation in severe trauma patients with bleeding shock. Resuscitation. 2010;81(7):804-9.

[6] Huh U, Song S, Chung SW, et al. Is extracorporeal cardiopulmonary resuscitation practical in severe chest trauma? A systematic review in single center of developing country. J Trauma Acute Care Surg. 2017;83(5):903-7.

[7] Swol J, Brodie D, Napolitano L, et al. Indications and outcomes of extracorporeal life support in trauma patients. J Trauma Acute Care Surg. 2018;84(6): 831-7.

[8] Zonies D, Merkel M. Advanced extracorporeal therapy in trauma. Curr Opin Crit Care. 2016;22(6):578-83.

[9] Grant AA, Hart VJ, Lineen EB, et al. The impact of an advanced ECMO program on traumatically injured patients. Artif Organs. 2018;42(11):1043-51.

[10] Hoops HE, Manning JE, Graham TL, et al. Selective aortic arch perfusion with fresh whole blood or HBOC201 reverses hemorrhage-induced traumatic cardiac arrest in a lethal model of noncompressible torso hemorrhage. J Trauma Acute Care Surg. 2019;87(2): 263-73. 
[11] Shishehbor MH, Moazami N, Tong MZ, et al. Cardiogenic shock: from ECMO to Impella and beyond. Cleve Clin J Med. 2017;84(4):287-95.

[12] Buck ML. Control of coagulation during extracorporeal membrane oxygenation. J Pediatr Pharmacol Ther. $2005 ; 10(1): 26-35$.

[13] Wikkelsø A, Wetterslev J, Møller AM, Afshari A. Thromboelastography (TEG) or thromboelastometry (ROTEM) to monitor haemostatic treatment versus usual care in adults or children with bleeding. Cochrane Database Syst Rev. 2016;22(8):CD007871.

[14] Lee N, Tang D, Jayaraman S. Penetrating cardiac trauma and the use of emergent extracorporeal membrane oxygenation and therapeutic hypothermia: when cooler heads prevail. Trauma Case Reports. 2015;1: 95-8. 